\title{
Effects of Age at Auditory Brainstem Implantation: Impact on Auditory Perception, Language Development, Speech Intelligibility
}

\author{
*Filiz Aslan, *Hilal Burcu Ozkan, *Esra Yücel, *Gonca Sennaroğlu, †Burçak Bilginer, \\ and $\ddagger$ Levent Sennaroğlu \\ *Audiology Department, Faculty of Health Sciences; †Neurosurgery Department; and $\ddagger$ Otorhinolaryngology Department, \\ Faculty of Medicine, Hacettepe University, Ankara, Turkey
}

\begin{abstract}
Objective: To study the effect of age at auditory brainstem implant (ABI) surgery on auditory perception, language, and speech intelligibility.

Study Design: Retrospective single cohort design.

Setting: Tertiary referral center.

Patients: In this study, 30 pediatric ABI users with no significant developmental issues were included. Participants were divided into two groups, according to age at surgery (Early Group: $<3$ yr old [ $=15]$, Late Group: $\geq 3$ yr old $[n=15])$. Groups were matched by duration of ABI use and participants were evaluated after 5 years $( \pm 1 \mathrm{yr})$ experience with their device. The mean age at ABI surgery was 22.27 (ranged \pm 6.5 ) months in the early group, 45.53 (ranged \pm 7.9 ) months in the late group.

Intervention(s): Retrosigmoid craniotomy and ABI placement.

Main Outcome Measure(s): Auditory perception skills were evaluated using the Meaningful Auditory Integration Scale and Categories of Auditory Performance from the Children's Auditory Perception Test Battery. We used a closed-set pattern perception subtest, a closed-set word identification subtest, and an open-set sentence recognition subtest. Lan-
\end{abstract}

Modern cochlear implant (CI) technologies have improved the auditory perception and language development skills of children with severe to profound sensorineural hearing loss (1). However, the benefit of CI has not reached the optimal level due to several variables, including age at implantation, mother's education level, early intervention approaches, residual hearing, and socioeconomic status (2-4). Another essential factor impacting CI success is anatomical malformation of the inner ear and/or auditory nerve $(5,6)$. Auditory brainstem implantation (ABI) is indicated as a viable option when

Address correspondence and reprint requests to Filiz Aslan, Ph.D., Department of Audiology, Faculty of Health Sciences, Hacettepe University, Ankara 16100, Turkey; E-mail: filizasl@gmail.com

The authors disclose no conflicts of interest.

DOI: $10.1097 /$ MAO.0000000000002455 guage performance was assessed with the Test of Early Language Development and Speech Intelligibility Rating, which was administered in a quiet room.

Results: In this study, the results demonstrated that the Early Group's auditory perception performance was better than the Late Group after 5 years of ABI use, when children had no additional needs $(\mathrm{U}=12, p<0.001)$. Speech intelligibility was the most challenging skill to develop, in both groups. Due to multiple regression analysis, we found that auditory perception categories can be estimated with speech intelligibility scores, pattern perception scores, receptive language scores, and age at $\mathrm{ABI}$ surgery variables in $\mathrm{ABI}$ users with no additional handicaps.

Conclusions: $\mathrm{ABI}$ is a viable option to provide auditory sensations for children with cochlear anomalies. ABI surgery under age 3 is associated with improved auditory perception and language development compared with older users. Key Words: Auditory brainstem implantsChildren-Rehabilitation.

Otol Neurotol 41:11-20, 2020. 
and as a result, only movements in and out of the recess were done according to eABR measurements. This part of the surgery is very important to avoid difficulties in mapping and the development of expected auditory perception skills. These risks and variables require experienced multidisciplinary teams in ABI surgery and in the fitting process (9).

Previous studies have shown that a multidisciplinary team approach is necessary to obtain better auditory and speech perception outcomes (10-12). A collaboration among neurotologists, neurosurgeons, pediatric audiologists, rehabilitative audiologists, teachers, special education professionals, and psychologists is important to the outcome. The professional assessment of the children from different perspectives determines realistic expectations after surgery. Realistic expectations help keep the parents' motivation higher in the rehabilitation process.

Auditory perception development in children with ABI is slower than that in CI users. Although the children with CI can still benefit from a different degree of residual hearing, ABI users with inner ear malformations (such as cochlear aplasia, cochlear nerve aplasia, etc.) cannot reach auditory stimulation until their first ABI mapping. Only children with acquired deafness involving temporal bone fracture, meningitis, NF2, or other such causes have the opportunity to develop listening skills before ABI surgery.

The negative effects of auditory deprivation on cochlear implantation have been presented in numerous studies $(4,13,14)$. Cochlear implantation not later than 4-years old and early intervention are essential for the development of speech perception, language development, and academic skills $(14,15)$. Currently, most centers implant children as young as 12 months and even younger $(16,17)$. ABI surgery in children is often performed later in life than with CI surgery. There are various reasons to delay the surgery, which also prolong auditory deprivation (i.e., surgical complications, developmental delays or additional health problems, inexperience of ABI team, lack of success with a cochlear implant etc.). In the preoperative period, it is advisable to diversify the communication options of the children and direct them to the sign language. Manual communication options (such as sign language, coded language, cued speech) are recommended to parents' of children with ABI to support their communication skills. It is observed that communication skills increase with the support of sign language, behavioral problems decrease, and provide children to follow the conversation with more than one person and in noisy environments.

In the early 2000s, Colletti et al. published the results of their studies with pediatric ABI users $(18,19)$, and their promising outcomes led professionals to explore ABI more fully. In Hacettepe University, Otorhinolaryngology Clinic, our team has worked with ABI users for more than a decade, and our aim is to determine the best approaches for improving our patients' quality of life.

In the present study, we aimed to examine the influence of age on auditory perception, language performance, and speech intelligibility skills of pediatric ABI users.

\section{METHODS}

This study was conducted at the Hacettepe University Department of Otorhinolaryngology, Audiology Unit. Having obtained the approval of the Ethical Committee for Non-Interventional Clinical Research of Hacettepe University (issue no. 16969557-1183 and decision no. NI 17/685), the data collection process was initiated.

\section{Participants}

The medical records of 100 pediatric ABI patients were reviewed, and 60 patients met the inclusion criteria. Of the 60 patients, 30 patients were matched for the duration of ABI use (Table 1). All implantations were performed by the same

TABLE 1. Demographical and audiological variables of children with auditory brainstem implants

\begin{tabular}{|c|c|c|c|c|c|c|c|}
\hline \multicolumn{8}{|c|}{ Children With Auditory Brainstem Implant $(\mathrm{n}=30)$} \\
\hline Categorical Variables & \multicolumn{2}{|c|}{ Early Group $n=15$} & $\%$ & \multicolumn{2}{|c|}{ Late Group $n=15$} & $\%$ & \multirow{2}{*}{$\frac{p \text { Value }}{0.77}$} \\
\hline Sex & & & & & & & \\
\hline Female & & & 67 & & & 73 & \\
\hline Male & & & 33 & & & 27 & \\
\hline ABI company & & & & & & & 0.37 \\
\hline Cochlear & & & 60 & & & 80 & \\
\hline Medel & & & 40 & & & 20 & \\
\hline Educational settings & & & & & & & 0.98 \\
\hline Special education & & & 80 & & & 67 & \\
\hline Mainstream & & & 13 & & & 20 & \\
\hline \multirow[t]{2}{*}{ School of Deaf } & & & 7 & & & 13 & \\
\hline & \multicolumn{3}{|c|}{ Early Group } & \multicolumn{3}{|c|}{ Late Group } & \\
\hline Continuous Variables & $\bar{X}$ & $S D$ & $N$ & $\bar{X}$ & SD & $\mathrm{n}$ & \\
\hline Age at evaluation (yr) & 7.07 & 0.96 & 15 & 9.60 & 1.4 & 15 & $0.001^{a}$ \\
\hline Age at ABI surgery (mo) & 22.27 & 6.5 & 15 & 45.53 & 7.9 & 15 & $0.001^{a}$ \\
\hline Duration of ABI use (yr) & 5.07 & 1.1 & 15 & 5.45 & 0.8 & 15 & 0.16 \\
\hline
\end{tabular}

\footnotetext{
${ }^{a} p<0.001$.
} 
neurotologist and neurosurgeon. The inclusion criteria for the study were as follows: 1) regular use of ABI (minimum $8 \mathrm{~h} / \mathrm{d}$ ), 2) for the Early Group, age at implantation $<3$ years old, 3) for the Late Group, age at implantation $\geq 3$ years old, 4) no other diagnosed additional needs, 5) children who resided in monolingual, Turkish-speaking homes, 6) unilateral ABI users who had at least 5 years of auditory experience with ABI and communicating verbally. In the Early Group, 10 of 15 children were female, and, in the late group, 11 of 15 children were female. All participants had congenital bilateral profound sensorineural hearing loss diagnosis and they did not benefit from hearing aids. Auditory rehabilitation services costs are funded by government in local special education centers and all participants enrolled the audiology department family consultation services regularly. All children prefer to use auditoryverbal or total communications methods.

Cochlear implant studies have also proved that additional needs (such as mental retardation, developmental delay, autism) diversely affect the outcomes in children $(20,21)$ and similarly delayed auditory and speech development can be observed in children with ABI (22). In this study, children with additional complex needs were excluded to rule out the known effects on the outcomes.

\section{Assessment Tools}

Both auditory perception and language development skills were assessed using standardized tests and questionnaires, which also represented the parents' perspectives. The assessment was administered in one session, taking 1 to 2 hours depending on the child. The parent questionnaires were completed by parents in the same day, before the assessment sessions.

\section{Auditory Perception}

The Categories of Auditory Performance-II (CAP II) was used to measure speech perception performance of the children with implants and was completed by either parents or clinicians $(23,24)$. The CAP II comprises a hierarchical scale of auditory perceptive ability and ranges from zero to seven: $0=$ no awareness of environmental sounds, $1=$ awareness of environmental sounds, $2=$ responds to speech sounds, $3=$ recognizes environmental sounds, $4=$ discriminates at least two speech sounds, $5=$ understands common phrases without lipreading, $6=$ understands conversation without lipreading with a familiar speaker, $7=$ can use the telephone with a familiar speaker (23).

The Auditory Integration Scale (MAIS) which is a parentreported questionnaire was used to assess listening skills in children with hearing loss. It consists of 10 items grouped into three main areas: implant use, awareness of sound, and sound recognition. The first two items concern the child's bonding with the device, including the child's willingness to wear it and his or her ability to recognize and identify device malfunctions. Items three to six relate to the child's alertness to sounds in everyday environments, and items seven through ten evaluate the child's ability to derive meaning from sound (20). Each item was rated by parents and scored from 0 to $4(0=$ never, $1=$ rarely, $2=$ occasionally, $3=$ frequently, and $4=$ always), with a total score ranging between 0 and 40 .

Speech perception subtests were administered both in closedand open-set conditions. Pattern perception, word identification, and sentence recognition skills were evaluated using, in the Turkish language, the Children's Auditory Perception Test Battery, which measures speech perception skills in three domains: 1) closed-set pattern perception, 2) closed-set word identification, and 3) open-set sentence recognition (21).
Closed-set pattern perception and closed-set word identification tests measured the children's abilities through two separate tasks. In the closed-set pattern perception test, 12 words with different numbers of syllables (one, two, or three syllables and compound words) were randomly presented, twice for each word, in auditory-only conditions, asking the child to point to or name the appropriate picture matching the word. If the child scored 17/24 or above, the researchers proceeded to the closed-set word identification test. This test aimed to determine the children's ability to identify words from a closed set of three-syllable words, hence assessing the ability of children with low language levels to use spectral information. Each word, as with the pattern perception test, was presented twice randomly. If the child scored 18/24 or above, the researchers proceeded to the open-set sentence recognition test. This test contained 6 lists of 10 sentences and attempted to assess children's ability to recognize and comprehend speech. The children were told they would hear a series of simple questions, statements, and commands, which they might have heard in their daily lives. Each sentence was presented only auditorily, using normal vocal effort. If, after the first five sentences, the child experienced obvious difficulty, to avoid distressing the child, we ended the test. All children were evaluated by the same list of sentences and a point was awarded for each word a child was able to correctly repeat. The total score was calculated as the number of words repeated as a percentage of the total number of words.

All speech perception tests were administered by the same female audiologist, using a live voice in auditory-only conditions. The speech stimuli were presented at a distance of $1 \mathrm{~m}$, at normal conversation volume, on the same side as the child's ABI.

\section{Language}

Language skills were assessed with the Test of Early Language Development-3 (TELD-3) (22). The test was administered in combined auditory-verbal conditions, and it included two subtests: receptive language and expressive language. TELD-3 assesses language development in children between the ages of 2 and 7 years, 11 months. Age equivalent scores are also reported for the subtests. The TELD-3 assesses a child's language development broadly, specifically in the areas of semantics, syntax, and morphology. In this study, total scores of receptive and expressive language subtests were used to compare the language performance between the two sample groups.

\section{Speech Intelligibility}

The Test of Early Language Development and Speech Intelligibility Rating (SIR) (25) was developed to measure speech intelligibility of children with ABI implants by quantifying their spontaneous speech for clinical comparisons. An experienced clinician rated each child's speech after listening to his or her connected speech. This was used to measure the child's speech intelligibility in real-life situations. SIR consists of five performance categories:

(1) Prerecognizable words in spoken language; the child's primary mode of everyday communication may be manual.

(2) Connected speech is unintelligible; intelligible speech is developing in single words when context and lipreading cues are available. 
(3) Connected speech is intelligible to a listener who concentrates and lipreads within a known context.

(4) Connected speech is intelligible to a listener who has little experience of a deaf person's speech; the listener does not need to concentrate unduly.

(5) Connected speech is intelligible to all listeners; the child is understood easily in everyday contexts.

MAIS, SIR, CAP II, and TELD-3 were administered by the same audiologist in a quiet therapy room without any visual or auditory distractions.

\section{Statistics}

All analyses were performed in the SPSS 20 statistics software (26). Continuous variables (i.e., duration of ABI use, chronological age, age at evaluation) were reported as means, standard deviations, and ranges. When the data from these tests was examined for normality and homogeneity of variance via the Shapiro-Wilk Test $(\mathrm{SW}=0.739, \mathrm{df}=30$, $p>0.001)$, the results suggested that the data was not distributed normally. A Q-Q plot and histogram gave similar results. Because of this, nonparametric tests (such as the Mann-Whitney $U$ test) were run across CAP Scores, MAIS scores, pattern perception scores, word identification scores, sentence recognition scores, language performance scores, and SIR scores. The differences among means with $p<0.05$ and $p<0.01$ accepted as representing statistically significant differences. Additionally, multiple regression analysis was conducted to examine direct and indirect relations among variables.

\section{RESULTS}

The chronological ages of the children at the time of evaluation were between 6 and 12 years old $(\mathrm{SD} \pm 6.6$ yrold). In both the Early and the Late Group, children were matched considering their duration of implant use. The mean age of ABI surgery in the Early Group was 22 months (ranged $12-33 \mathrm{mo}, \pm 7$ ), and, for the Late Group, it was 46 months (ranged $36-64 \mathrm{mo}, \pm 8$ ), as shown in Table 1.

\section{Auditory Perception Outcomes}

All participants had achieved auditory sensation and used their ABI eagerly. In both groups, children developed basic auditory perception skills, such as recognizing environmental sounds and responding to their names. In the Early Group, the mean score for CAP II was 5 $(\min =4$ and $\max =6, \mathrm{SD} \pm 0.79)$, and, in the Late Group, it was $3(\min =2$ and $\max =4, \mathrm{SD} \pm 0.92)$. According to the Mann-Whitney $U$ test, there was a statistically significant difference between the Early Group and the Late Group in CAP II scores $(\mathrm{U}=12$, $p<0.001)$. This result suggests that most of the children in the Early Group started to understand common phrases without lipreading, while most of the children in the Late Group only identified environmental sounds by the end of approximately 5 years (range between 4 and $6 \mathrm{yr}$ ).

In the Early Group, the mean MAIS score was 37 $(\min =30$ and $\max =40, \mathrm{SD} \pm 3.61)$, while it was 25 $(\min =5$ and $\max =40, \mathrm{SD} \pm 13.68)$ in the Late Group. This outcome indicates that there was, again, a statistically significant difference between the two groups
$(\mathrm{U}=64.50, p<0.045)$ (Fig. 1). The Early Group had better, meaningful auditory integration skills in everyday situations when compared with the Late Group. In addition to the total MAIS scores of both groups, the item scores from the questionnaires were examined. It was found that those who had implantation surgery before age 3 , and those who underwent it after age 3 , had similar scores in the first two items, which questioned the child's bonding with the device, including willingness to wear it. However, when the scores of items three through six were examined, it was found that the Late Group had lower scores than the Early Group. This indicates that the Late Group was less alert to sounds in everyday environments. The Late Group also showed poorer performance on deriving meaning from sound, as measured by items 7 through 10 (Fig. 1).

Pattern perception scores also demonstrated the advantage of ABI surgery at early stages of life. Out of 15 children in the Early Group, 14 were able to complete the task scoring 24/24 (100\%). Only one child scored 22/24 (92\%) but also passed the criteria for continuing on to the word identification testing. In the Late Group, only 4 of 15 children could complete the test and pass the criteria with 24/24 (100\%). Six children had scores between 14/ 24 and $23 / 24$, but only five of them passed the criteria for continuing to the next research stage. The test was not administered to five children from the Late Group, as the task was too difficult for them to carry out (Table 2). The Mann-Whitney $U$ test indicated that pattern perception percentage scores were higher in the Early Group $(\mathrm{Mdn}=100)$ when compared with the Late Group $(\mathrm{Mdn}=87, \mathrm{U}=37, p<0.001)$ (Table 3).

Concerning the closed-set word identification test, in the Early Group, 9 of 15 children were able to complete the task scoring $24 / 24(100 \%)$. Three children scored 18/ $24(75 \%)$, but also passed the criteria for having mastered word identification enough to continue on to the open-set sentence recognition test. Only three children could not pass the criteria, and they had scores between 8 and 14. In the Late Group, 2 of 15 children completed the test and passed the criteria scoring 24/24 (100\%). Seven children had scores between 14/24 and 23/24, and six of them passed the criteria for continuing. The test was not administered to six children, for whom the task was too difficult (Table 2). This result indicated that the Early Group had better scores for word identification $(\mathrm{Mdn}=100)$ than the Late Group $(\mathrm{Mdn}=83, \mathrm{U}=59$, $p=0.026$ ) (Table 3).

The sentence recognition test was also administered to evaluate the open-set speech understanding of each group. In the Early Group, 8 of 15 children had progressed enough to recognize and repeat the everyday sentences during the live voice testing. Six children had scores ranging from 10 to 40 , and they could not pass the required criteria, which indicated they still continued to progress. Only one child was not ready for testing in this group. In the Late Group, five children passed the criteria, and they scored between 6 and 8 . The rest of the children were not able to continue testing (Table 2). In the 


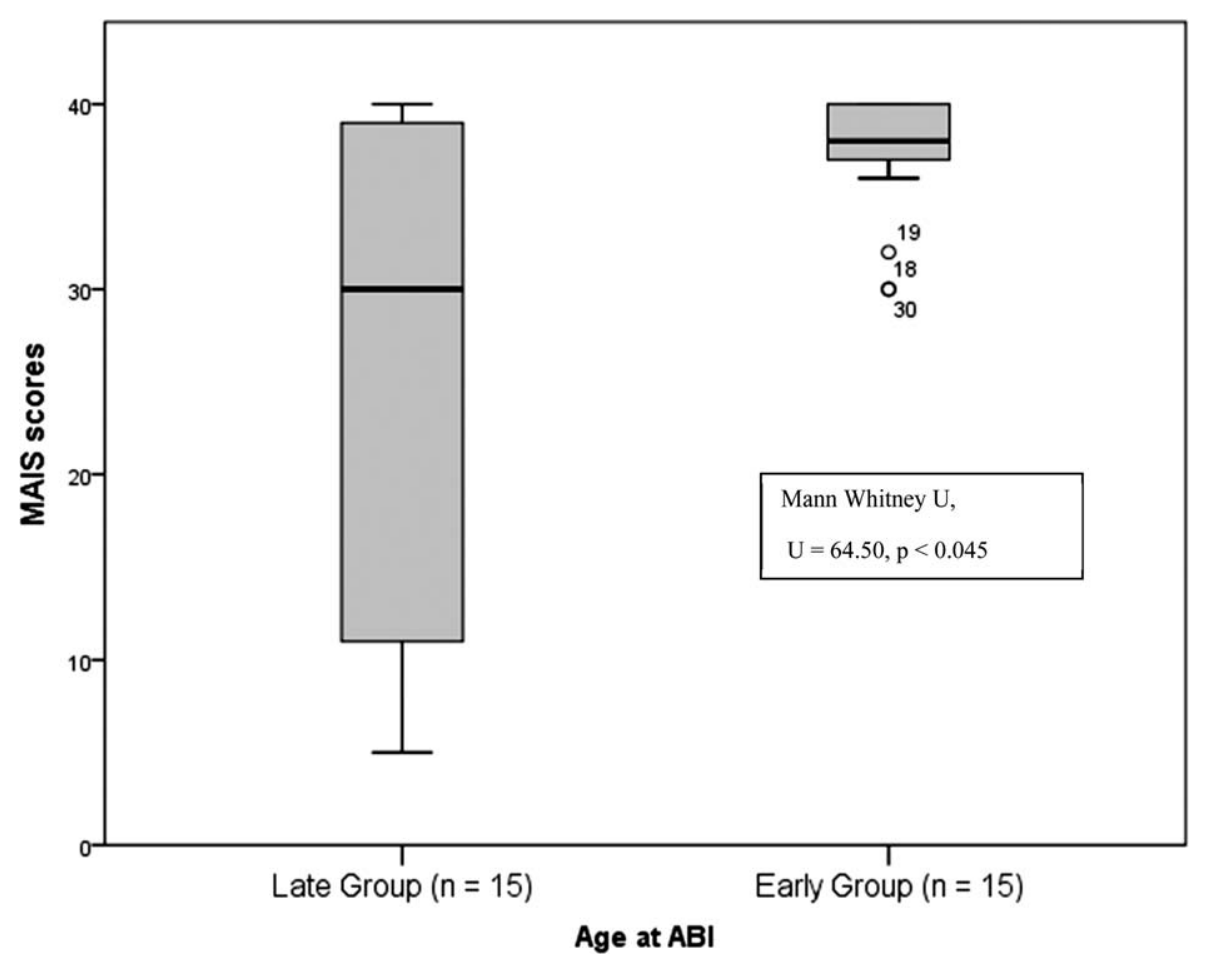

FIG. 1. Comparison of Meaningful Auditory Integration Scale (MAIS) scores between early (implanted under $3 \mathrm{yr}$ of age) and late (implanted at or beyond $3 \mathrm{yr}$ of age) Auditory Brainstem Implant groups.

Mann-Whitney $U$ test, the Early Group $(\operatorname{Mdn}=50)$ and the Late Group ( $\mathrm{Mdn}=0)$ showed statistically significant differences in the sentence recognition task $(\mathrm{U}=71.50$, $p=0.09$ ) (Table 3).

The results of auditory perception, speech perception, and speech intelligibility are shown in detail in Table 4.

\section{Language and Speech Intelligibility Performance}

Language performance was assessed with TELD-3 in two subtests, and scores were analyzed based on ageequivalent scores. The Shapiro-Wilk analysis showed that receptive language scores were probably distributed normally $(\mathrm{SW}=0.95, \mathrm{df}=30, p=0.18)$. An Independent Sample $t$ test indicated that receptive language scores were similar between the Early Group $(\mathrm{M}=41.80, \quad \mathrm{SD}=10.85)$ and the Late Group $(\mathrm{M}=35.67, \mathrm{SD}=13.69), t(28)=1.36, p>0.05$. Children's language comprehension skills were evaluated by auditory verbal mode. Age-equivalent scores were used to evaluate the scores as there were no standard scores consistent with chronological ages. According to the results, when the age at which they began to use ABI is perceived as "hearing age" rather than chronological age of the children, it is observed that the early group shows close to the hearing ages (Fig. 2).

The second subtest of language development was expressive language subtest and its scores were not normally distributed $(\mathrm{SW}=0.91, \mathrm{df}=30, p<0.05)$. The Mann-Whitney $U$ test showed that expressive language performance of the Early Group $(\mathrm{Mdn}=30)$ and the Late Group $(\mathrm{Mdn}=29)$ were similar $(\mathrm{U}=88$, $p=0.30$ ). As there were no standard scores compatible with chronological ages for the expressive language scores, the groups were compared with age-equivalence scores. It was evaluated verbally. There was no significant difference between the two groups. According to these results, both groups have difficulties in expressive language skills (Fig. 2).

Speech intelligibility scores were also analyzed with the Mann-Whitney $U$ test, and the results indicated that speech intelligibility performance of the Early Group $(\mathrm{Mdn}=3)$ was better than that of the Late Group $(\mathrm{Mdn}=1, \mathrm{U}=30, p<0.001)$ (Fig. 3). These results suggest that most of the children in the Early group reached level three ("Connected speech is intelligible to a listener who concentrates and lipreads"), while the Late Group stayed at level one ("Pre-recognizable words in spoken language; the child's primary mode of everyday communication may be manual. ").

\section{A Model Suggestion to Improve the Auditory Perception Outcomes}

Following the direction of these findings, we wanted to investigate which variables primarily predicted the auditory perception performance of ABI users. We used CAP II scores as a predictor of auditory perception performance, which includes everyday listening performance and auditory perception development. We hypothesized that age at ABI surgery, receptive language performance, closed-set pattern perception scores, and speech intelligibility scores would predict the CAP II scores. The Enter method of multiple linear regression analysis showed that 
TABLE 2. Individual raw scores and percentage scores of speech perception tests

\begin{tabular}{|c|c|c|c|c|c|c|c|c|c|c|}
\hline & & $\begin{array}{l}\text { Pattern Discrimination } \\
(\mathrm{TS}=24)\end{array}$ & $\%$ & $\begin{array}{l}\text { Pass Criteria } \\
(17 / 24)\end{array}$ & $\begin{array}{l}\text { Word Identification } \\
(\mathrm{TS}=24)\end{array}$ & $\%$ & $\begin{array}{l}\text { Pass Criteria } \\
(18 / 24)\end{array}$ & $\begin{array}{l}\text { Sentence Recognition } \\
(\mathrm{TS}=10)\end{array}$ & $\%$ & $\begin{array}{l}\text { Pass Criteria } \\
(>50 \%)\end{array}$ \\
\hline \multirow[t]{15}{*}{ Late group } & 1 & 0 & 0 & Fail & 0 & 0 & Fail & 0 & 0 & Fail \\
\hline & 2 & 0 & 0 & Fail & 0 & 0 & Fail & 0 & 0 & Fail \\
\hline & 3 & 24 & 100 & Pass & 24 & 100 & Pass & 8 & 80 & Pass \\
\hline & 4 & 0 & 0 & Fail & 0 & 0 & Fail & 0 & 0 & Fail \\
\hline & 5 & 0 & 0 & Fail & 0 & 0 & Fail & 0 & 0 & Fail \\
\hline & 6 & 24 & 100 & Pass & 20 & 83 & Pass & 7 & 70 & Pass \\
\hline & 7 & 14 & 59 & Fail & 0 & 0 & Fail & 0 & 0 & Fail \\
\hline & 8 & 24 & 100 & Pass & 24 & 100 & Pass & 0 & 0 & Fail \\
\hline & 9 & 23 & 95 & Pass & 22 & 92 & Pass & 9 & 90 & Pass \\
\hline & 10 & 20 & 83 & Pass & 20 & 83 & Pass & 6 & 60 & Pass \\
\hline & 11 & 22 & 92 & Pass & 14 & 59 & Fail & 0 & 0 & Fail \\
\hline & 12 & 0 & 0 & Fail & 0 & 0 & Fail & 0 & 0 & Fail \\
\hline & 13 & 24 & 100 & Pass & 22 & 92 & Pass & 8 & 80 & Pass \\
\hline & 14 & 23 & 95 & Pass & 22 & 92 & Pass & 0 & 0 & Fail \\
\hline & 15 & 23 & 95 & Pass & 22 & 92 & Pass & 0 & 0 & Fail \\
\hline \multirow[t]{15}{*}{ Early group } & 16 & 24 & 100 & Pass & 14 & 59 & Fail & 6 & 60 & Pass \\
\hline & 17 & 24 & 100 & Pass & 18 & 75 & Pass & 8 & 80 & Pass \\
\hline & 18 & 24 & 100 & Pass & 24 & 100 & Pass & 4 & 40 & Fail \\
\hline & 19 & 24 & 100 & Pass & 14 & 59 & Fail & 1 & 10 & Fail \\
\hline & 20 & 24 & 100 & Pass & 18 & 75 & Pass & 3 & 30 & Fail \\
\hline & 21 & 24 & 100 & Pass & 24 & 100 & Pass & 4 & 40 & Fail \\
\hline & 22 & 24 & 100 & Pass & 24 & 100 & Pass & 6 & 60 & Pass \\
\hline & 23 & 24 & 100 & Pass & 24 & 100 & Pass & 5 & 50 & Pass \\
\hline & 24 & 24 & 100 & Pass & 24 & 100 & Pass & 5 & 50 & Pass \\
\hline & 25 & 24 & 100 & Pass & 24 & 100 & Pass & 4 & 40 & Fail \\
\hline & 26 & 24 & 100 & Pass & 24 & 100 & Pass & 3 & 30 & Fail \\
\hline & 27 & 24 & 100 & Pass & 24 & 100 & Pass & 7 & 70 & Pass \\
\hline & 28 & 22 & 92 & Pass & 18 & 75 & Pass & 5 & 50 & Pass \\
\hline & 29 & 24 & 100 & Pass & 24 & 100 & Pass & 8 & 80 & Pass \\
\hline & 30 & 24 & 100 & Pass & 8 & 33 & Fail & 0 & 0 & Fail \\
\hline
\end{tabular}

TS indicates total score.

a significant regression equation was found $(\mathrm{F}=(4$, $25)=29.87, p<0.001)$, with $\mathrm{R}^{2}$ of .83 . Participants' predicted CAP II scores are equal to 2.49 $0.02 \times($ Age at $\mathrm{ABI}$ surgery $)+0.77 \times($ SIR scores $)+0.1$ $\times$ (Pattern Perception scores) $-0.1 \times$ (Receptive Language Scores). Adjusted $\mathrm{R}^{2}$ indicated that this model estimated $80 \%$ of the auditory perception scores in the pediatric ABI population. This model also showed that speech intelligibility, pattern perception performance, receptive language performance, and age at ABI surgery were highly related to auditory perception categories and can be used to estimate auditory perception performance.

\section{DISCUSSION}

In this study, we aimed to investigate the effects of age at $\mathrm{ABI}$ on auditory perception, language performance, and speech intelligibility on children with no additional needs. The results of this study indicated three main findings: 1) the children who received ABI under 3-years old performed better on auditory perception than the children who received ABI after 3-years old, 2) language performance scores were not significantly different between groups, 3) speech intelligibility scores were better in the early group than the late group. Regarding

TABLE 3. $t$ test results of two groups in speech perception tests

\begin{tabular}{|c|c|c|c|c|c|c|c|c|c|c|c|c|}
\hline & \multicolumn{4}{|c|}{ Pattern Perception } & \multicolumn{4}{|c|}{ Word Identification } & \multicolumn{4}{|c|}{ Sentence Recognition } \\
\hline & $\begin{array}{l}\text { Mean } \\
\text { Rank }\end{array}$ & $\begin{array}{l}\text { Sum of } \\
\text { Ranks }\end{array}$ & $\begin{array}{c}Z \\
\text { Score }\end{array}$ & $p$ & $\begin{array}{l}\text { Mean } \\
\text { Rank }\end{array}$ & $\begin{array}{c}\text { Sum of } \\
\text { Ranks }\end{array}$ & $\begin{array}{c}Z \\
\text { Score }\end{array}$ & $p$ & $\begin{array}{l}\text { Mean } \\
\text { Rank }\end{array}$ & $\begin{array}{l}\text { Sum of } \\
\text { Ranks }\end{array}$ & $\begin{array}{c}Z \\
\text { Score }\end{array}$ & $p$ \\
\hline The Early group $(n=15)$ & 20.53 & 308 & -3.55 & $0.001^{a}$ & 19.07 & 286 & -2.29 & $0.026^{b}$ & 18.23 & 273.50 & -1.75 & 0.080 \\
\hline The late group $(n=15)$ & 10.47 & 157 & & & 11.93 & 179 & & & 12.77 & 191.50 & & \\
\hline
\end{tabular}

$$
\begin{aligned}
& { }_{b}^{a}<0.001 . \\
& { }_{p}<0.05
\end{aligned}
$$


TABLE 4. Both groups' performances on auditory perception, speech perception and speech intelligibility

\begin{tabular}{|c|c|c|c|c|c|}
\hline & \multicolumn{2}{|c|}{ Early Group $(\mathrm{n}=15)$} & \multicolumn{3}{|c|}{ Late Group $(\mathrm{n}=15)$} \\
\hline & Mean & Standard Deviation & Mean & Standard Deviation & $p$ Values \\
\hline Categories of auditory performance & 5 & 0.79 & 3 & 0.92 & $0.001^{b}$ \\
\hline Meaningful auditory Integration scale & 37 & 3.61 & 25 & 13.68 & $0.045^{a}$ \\
\hline Pattern perception scores & 23.87 & 0.52 & 12.67 & 11.07 & $0.001^{b}$ \\
\hline Word identification scores & 20.40 & 5.14 & 12.67 & 10.95 & $0.026^{a}$ \\
\hline Sentence recognition scores & 46 & 22.93 & 25.33 & 37.59 & 0.089 \\
\hline Speech intelligibility scores & 3 & 1.10 & 1.40 & 0.51 & $0.001^{b}$ \\
\hline
\end{tabular}

$$
\begin{aligned}
& { }_{p}^{a}<0.05 \\
& { }_{p}<<0.01
\end{aligned}
$$

their speech perception performances, for a majority of the children in the Early Group, it was possible to evaluate the ability to define open-set sentence recognition skills because they passed the closed-set pattern and word identification levels. However, in the sentence recognition task, those children still needed visual cues for assistance (i.e., lip-reading, gestures). Although Early
Group was performed better on speech perception tasks, a few children could not reach their peers. Similar trending can be seen in the Late Group, most of the children found the speech perception tasks challenging some children performed similar to their Early Group peers. These results should be examined and outcomes interpreted cautiously. Because in this study, variables such as

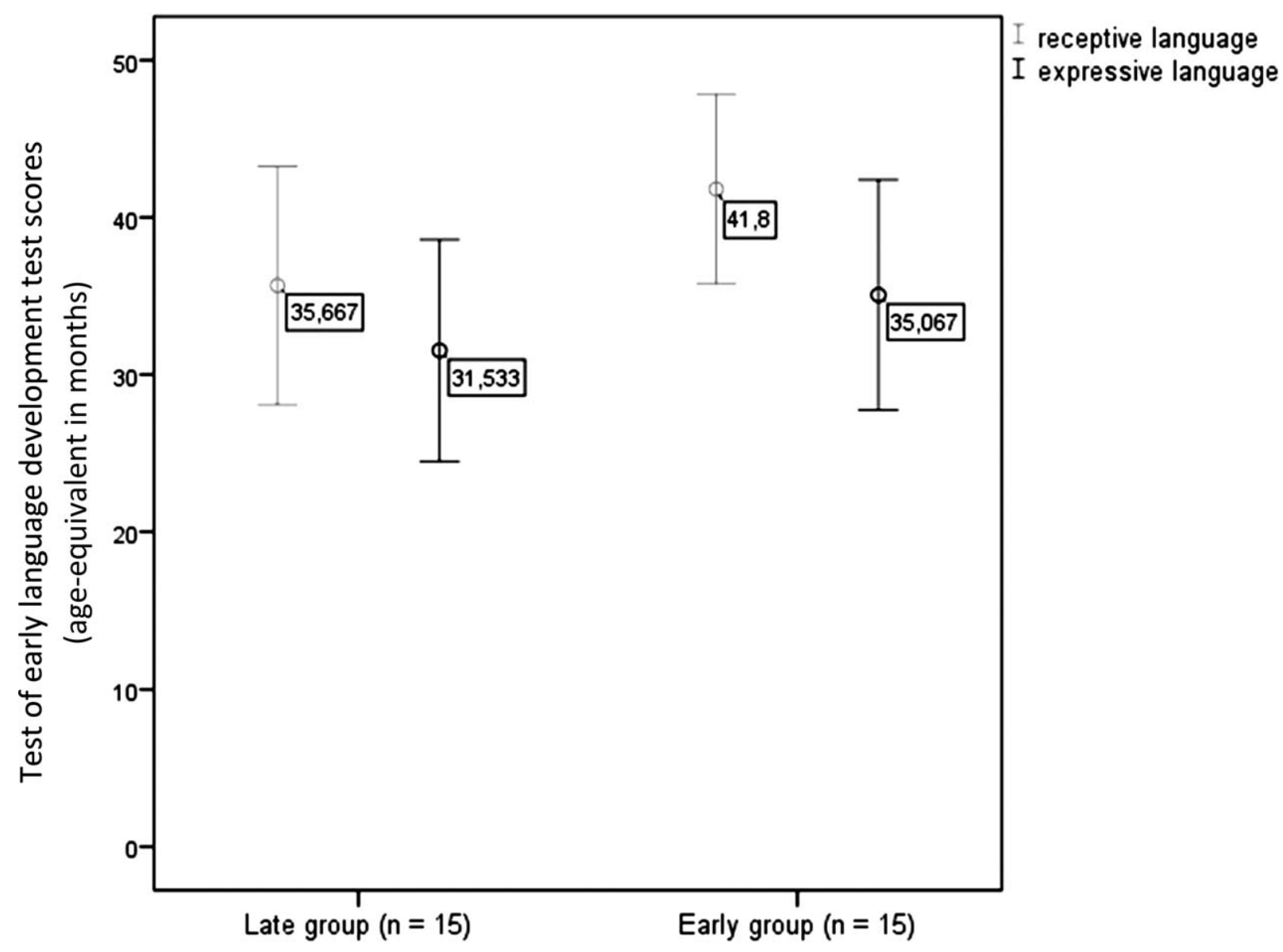

Age at $A B I$ surgery

FIG. 2. Comparison in receptive and expressive language performances between early (implanted under 3 yr of age) and late (implanted at or beyond $3 \mathrm{yr}$ of age). 


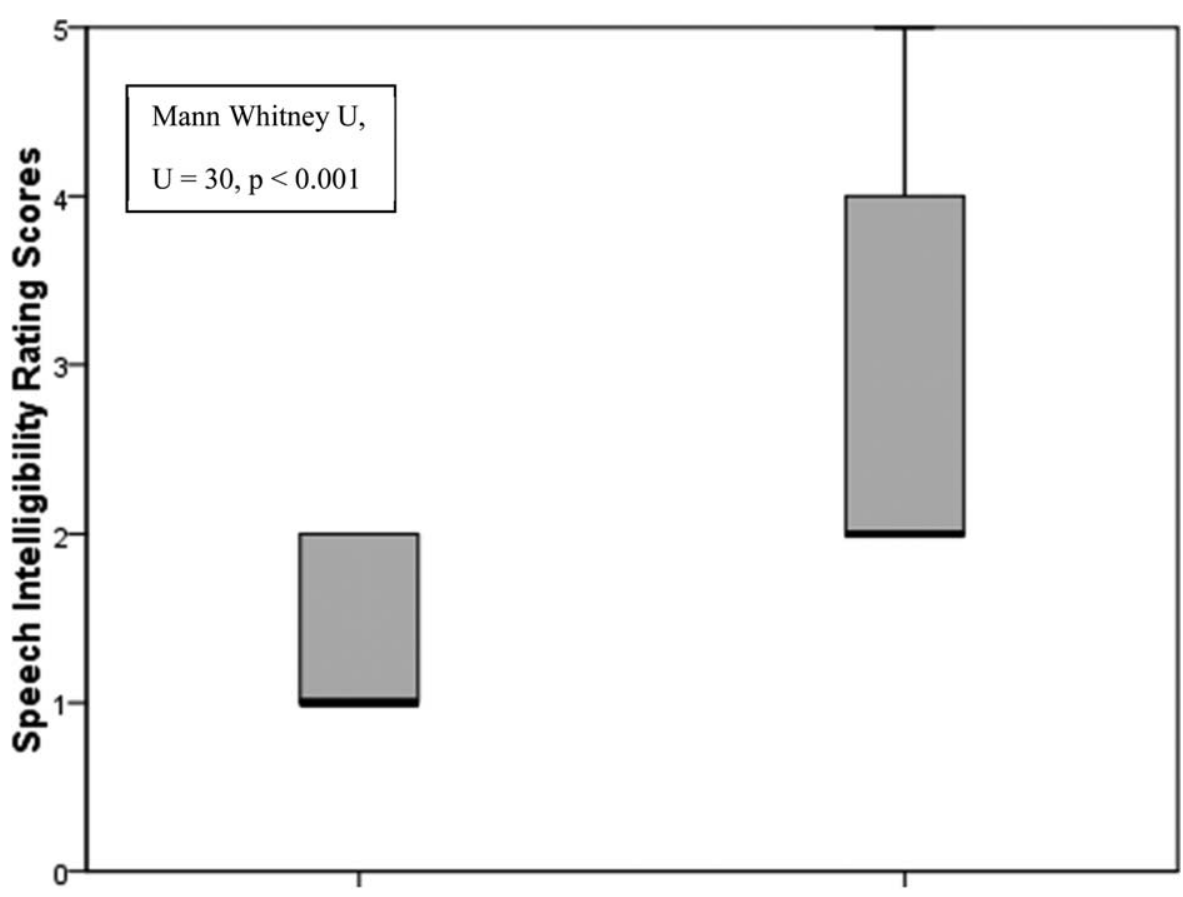

Late Group ( $\mathrm{n}=15) \quad$ Early Group $(\mathrm{n}=15)$

FIG. 3. Speech intelligibility rating (SIR) scores of early group (implanted under $3 \mathrm{yr}$ of age) and late group (implanted at or beyond $3 \mathrm{yr}$ of age).

parents' education leveles, socioeconomic status of the family were not included in the analysis. In future studies, it is recommended that the effects of these variables can be included in the evaluation to establish more precise findings.

In fact, children who use ABI evidence a distinct difference in their auditory skills at the end of the first year (27-29). Taking these results into account, in our study only the children who had auditory experience with their ABI for more than 5 years were included to eliminate the possible effects of adaptation processes. Da costa Monsanto et al. (30) reported that a great portion of children with ABI (93\% across 24 studies) started to detect environmental sounds and develop speech perception ability. These results correlate with our findings, as all children, irrespective of age at implantation, could achieve detection and reorganization skills for environmental and speech sounds.

Children's daily listening skills were evaluated by MAIS and the information was collected from their parents. The Early Group had better scores than Late Group in MAIS total score. When the MAIS scores examined in detail, it was determined that the late group frequently had better scores from 1 and 4 which are evaluated the sound awareness and identification. These findings suggest that the Late Group might achieve similar scores to the Early Group in sound awareness skills, but they had lower scores on items that included sound identification and open-set speech perception skills in MAIS. These results were consistent with those obtained from tests in closed-set and open-set conditions. In the previous literature, MAIS scores of children with ABI were frequently given as total scores, but no study reported the test items that assessed the device use and the auditory perception skills in detail. However, in studies with children using cochlear implants, rapid progress was observed in the MAIS subtests after implantation (20,31). Although the MAIS scores of children who underwent $\mathrm{ABI}$ under the age of 3 were significantly higher, it was found that three children received better scores than the late implant group but lower than their groups. Due to this result, early surgery is not sufficient and it is recommended to consider the variables such as family participation, educational environment, and socioeconomic level. Since these variables are not included in this study, it is aimed to increase the studies in this respect in the future.

In the Early Group, the youngest child underwent ABI surgery at 12 months old and the oldest at 33 months. Considering the CAP II scores, children in the Early Group performed better-similarly to their peers using CIs - and had higher CAP II scores than the children who had their ABI surgeries after they turned 3 years old (32). Although many of the children in the Early Group started to recognize common phrases (CAP II Category 5), children in the Late Group were mostly at the speech sound identification level (CAP II Category 3). Studies on the effects of early cochlear implantation (before users reaching 3 yrs old) have shown that such users develop speech recognition skills nearly in the first 2 years after 
implantation $(33,34)$. Typically developing children with CIs do not need to rely on visual cues, in contrast to children with ABI $(35,36)$. Although both Early and Late Groups relied on lip-reading or speech-reading, the Early Group required fewer visual cues in this study.

Another developmental domain in this study was speech intelligibility, which was measured via SIR. In this article, children in the Early Group also performed better in speech intelligibility than children in the Late Group. The results we obtained from both the Early and the Late Groups were lower than results from previous CI studies $(37,38)$. De Raeve et al.'s study has been studied as an example in that it emphasized the importance of early cochlear implantation and showed rapid development of speech intelligibility in the first 3 years, although there are significant differences with the current study (39). In their study, 52 children who received their CIs before 18 months old were evaluated in the 6th, 12th, 24th, and 36th months after surgery. Their SIR scores improved from intelligible speech in single words when context and lip-reading cues were available (SIR Category 2 , after $1 \mathrm{yr}$ ) to their connected speech being intelligible to a known listener who concentrates and lip-reads (SIR Category 3, after 2 yr). At their third-year interval, the median score of SIR progressed to their speech being intelligible to a listener who has little experience with deaf people (SIR Category 4). High pitched sounds are essential to speech intelligibility and speech perception in sentences (40). Choi et al.'s latest study evidenced that nontumor patients with $\mathrm{ABI}$ had limitations in high pitched sounds, such as $/ \mathrm{sh} /$ and $/ \mathrm{s} /$ (41). Additionally, there is still not enough information about how ABI users process acoustical cues. It is known that frequency tonotopicity of the cochlear nucleus is not organized at the surface, but is, rather, in subunits and layers $(42,43)$. When $A B I$ is placed on the surface of the cochlear nucleus, the process of temporal and spectral resolution afterward is unknown. For all that, some children do develop intelligible speech with $\mathrm{ABI}$ during the rehabilitation process. Further studies are needed to determine if there is a direct effect between speech intelligibility and high-pitched sound perception in ABI users.

The difference between chronological age and language performance is similar to that found by other studies $(29,44)$ Both groups showed similar scores on language test items, indicating that they had not fully progressed and they needed to integrate auditory perception and language skills. Despite the superiority of speech perception scores in the Early Group, their receptive and expressive language scores were not significantly different than Late Group. These results may lead to indirect effect on higher cognitive skills (memory, verbal reasoning, and problem solving). Additionally, the complexity of the language tests increased with age, and children with $A B I$ need more time to develop better language skills than do CI users. If further studies gather more children, and follow the language skills of these children progressing over time, the subtests' performance may be evaluated comprehensively, and it can then be determined which skills are superior and which skills are challenging.

In this study, it was found that auditory perception categories can be estimated with speech intelligibility scores, pattern perception scores, receptive language scores, and age at ABI surgery variables. Thus, clinics with less pediatric $\mathrm{ABI}$ experience can use this formula to determine the possible category children with no additional needs will fall into, after follow-up processes, and, therefore, inform the parents about what to expect after surgery.

This study has several limitations, first the sample size was limited. ABI is a fairly new procedure, and it is not as widespread as CI. Second, the participants represented a narrow range of ages and $\mathrm{ABI}$ users. Including children with additional needs, and who had less than 5 years of ABI use, could have diversified the rehabilitation outcomes because the children who need ABI often have additional needs. The results may not be generalized to the all pediatric $\mathrm{ABI}$ recipients due to sampling limitation and exclusion of children with additional needs. Professionals should also include multisensory integration therapies in their individualized programs (45). For example, the patients' attention must be assessed and supported - their attention spans, their ability to sustain attention in a conversation, and their ability to select auditory information from other distractions.

In the future studies, increasing the sample size, rather than conducting focus groups, may provide greater depth of information. Additionally, variables such as parents' education levels, parents' ages, and the education quality of local rehabilitation centers could be assessed to show their impacts on auditory perception and language outcomes.

Additionally, even children who did not perform as well as other participants still use their ABIs regularly. In this case, we should consider benefits of $\mathrm{ABI}$ in children more broadly, because our aim is to support their life quality and communication skills. During the decision to surgery, surgeons and audiologists can consider that the use of ABI under 3 years old may have positive effects, when a child is an eligible candidate.

To the authors' knowledge, this is the first study to examine the effects of age at $\mathrm{ABI}$ on auditory perception, language, and speech intelligibility. It is known that auditory deprivation negatively affects language development $(46,47)$. In the ABI candidacy selection process, the challenges presented here should be kept in mind, and expectations from $\mathrm{ABI}$ must be shaped within this context.

\section{REFERENCES}

1. Mellon NK. Language and Speech Acquisition, 2nd ed. USA: Lippincott Williams \& Wilkins; 2009.

2. Niparko JK, Tobey EA, Thal DJ, et al. Spoken language development in children following cochlear implantation. JAMA 2010; 303:1498-506.

3. Geers A, Brenner C, Davidson L. Factors associated with development of speech perception skills in children implanted by age five. Ear Hear 2003;24:24S-35S. 
4. Tobey EA, Thal D, Niparko JK, et al. Influence of implantation age on school-age language performance in pediatric cochlear implant users. Int J Audiol 2013;52:219-29.

5. Buchman CA, Copeland BJ, Yu KK, Brown CJ, Carrasco VN, Pillsbury HC III. Cochlear implantation in children with congenital inner ear malformations. Laryngoscope 2004;114:309-16.

6. Pakdaman MN, Herrmann BS, Curtin HD, Van Beek-King J, Lee DJ. Cochlear implantation in children with anomalous cochleovestibular anatomy: A systematic review. Otolaryngol Head Neck Surg 2012;146:180-90.

7. Sennaroglu L, Sennaroglu G, Yücel E, et al. Long-term results of $\mathrm{ABI}$ in children with severe inner ear malformations. Otol Neurotol 2016;37:865-72.

8. Colletti V, Shannon RV. Open set speech perception with auditory brainstem implant? Laryngoscope 2005;115:1974-8.

9. Wilkinson EP, Eisenberg LS, Krieger MD, et al. Initial results of a safety and feasibility study of auditory brainstem implantation in congenitally deaf children. Otol Neurotol 2017;38:212-20.

10. Sennaroglu L, Colletti V, Manrique M, et al. Auditory brainstem implantation in children and non-neurofibromatosis type 2 patients: A consensus statement. Otol Neurotol 2011;32:187-91.

11. Sennaroglu L, Ziyal I. Auditory brainstem implantation. Auris Nasus Larynx 2012;39:439-50.

12. Buchman CA, Teagle HF, Roush PA, et al. Cochlear implantation in children with labyrinthine anomalies and cochlear nerve deficiency: Implications for auditory brainstem implantation. Laryngoscope 2011;121:1979-88.

13. Houston DM, Miyamoto RT. Effects of early auditory experience on word learning and speech perception in deaf children with cochlear implants: Implications for sensitive periods of language development. Otol Neurotol 2010;31:1248-53.

14. Sharma A, Dorman MF, Spahr AJ. A sensitive period for the development of the central auditory system in children with cochlear implants: Implications for age of implantation. Ear Hear 2002;23:532-9.

15. Sharma A, Dorman MF, Kral A. The influence of a sensitive period on central auditory development in children with unilateral and bilateral cochlear implants. Hear Res 2005;203:134-43.

16. Leigh J, Dettman S, Dowell R, Briggs R. Communication development in children who receive a cochlear implant by 12 months of age. Otol Neurotol 2013;34:443-50.

17. Waltzman SB, Roland JT. Cochlear implantation in children younger than 12 months. Pediatrics 2005;116:487-93.

18. Colletti V, Carner M, Miorelli V, Guida M, Colletti L, Fiorino F. Auditory brainstem implant (ABI): New frontiers in adults and children. Otolaryngol Head Neck Surg 2005;133:126-38.

19. Colletti V, Carner M, Fiorino F, et al. Hearing restoration with auditory brainstem implant in three children with cochlear nerve aplasia. Otol Neurotol 2002;23:682-93.

20. Robbins AM, Renshaw JJ, Berry SW. Evaluating meaningful auditory integration in profoundly hearing-impaired children. $\mathrm{Am}$ J Otol 1991;12:144-50.

21. Yucel E, Sennaroglu G. Auditory Perception Test Battery for Children. 2011.

22. Güven S, Topbas? S. Adaptation of the test of Early Language Development-(TELD-3) into Turkish: Reliability and Validity Study. Int J Early Childhood Special Educ 2014;6:151-76.

23. Gilmour L. The inter-rater reliability of categories of auditory performance-II (CAP)-II. University of Southampton; 2010.

24. Archbold S, Lutman M, Marshall D. Categories of auditory performance. Ann Otol Rhinol Laryngol Suppl 1995;166:312-4.

25. Cox RM, McDaniel DM. Development of the Speech Intelligibility Rating (SIR) test for hearing aid comparisons. J Speech Hear Res 1989;32:347-52.

26. SPSS I. IBM SPSS statistics for Windows, version 20.0. New York: IBM Corp; 2011.
27. Colletti V, Fiorino F, Sacchetto L, Miorelli V, Carner M. Hearing habilitation with auditory brainstem implantation in two children with cochlear nerve aplasia. Int $J$ Pediatr Otorhinolaryngol 2001;60:99-111.

28. Nevison B, Laszig R, Sollmann W-P, et al. Results from a European clinical investigation of the Nucleus $(\mathbb{R}$ multichannel auditory brainstem implant. Ear Hear 2002;23:170-83.

29. Eisenberg LS, Johnson KC, Martinez AS, et al. Comprehensive evaluation of a child with an auditory brainstem implant. Otol Neurotol 2008;29:251-7.

30. da Costa Monsanto R, Bittencourt AG, Neto NJB, et al. Auditory brainstem implants in children: Results based on a review of the literature. Int Adv Otol 2014;10:284-90.

31. Zhong Y, Xu T, Dong R, Lyu J, Liu B, Chen X. The analysis of reliability and validity of the IT-MAIS, MAIS and MUSS. Int $J$ Pediatr Otorhinolaryngol 2017;96:106-10.

32. Govaerts PJ, De Beukelaer C, Daemers K, et al. Outcome of cochlear implantation at different ages from 0 to 6 years. Otol Neurotol 2002;23:885-90.

33. Colletti V, Carner M, Miorelli V, Guida M, Colletti L, Fiorino FG. Cochlear implantation at under 12 months: Report on 10 patients. Laryngoscope 2005;115:445-9.

34. Colletti L, Mandalà M, Zoccante L, Shannon RV, Colletti V. Infants versus older children fitted with cochlear implants: Performance over 10 years. Int J Pediatr Otorhinolaryngol 2011;75:504-9.

35. Bergeson TR, Pisoni DB, Davis RA. Development of audiovisual comprehension skills in prelingually deaf children with cochlear implants. Ear Hear 2005;26:149-64.

36. Bergeson TR, Houston DM, Miyamoto RT. Effects of congenital hearing loss and cochlear implantation on audiovisual speech perception in infants and children. Restor Neurol Neurosci 2010;28:157-65.

37. Montag JL, AuBuchon AM, Pisoni DB, Kronenberger WG. Speech intelligibility in deaf children after long-term cochlear implant use. $J$ Speech Lang Hear Res 2014;57:2332-43.

38. Schafer E, Utrup A. The effect of age of cochlear implantation on speech intelligibility to others. J Educ Pediatr (Re) Habilitative Audiol 2016;22:51-61.

39. De Raeve L. A longitudinal study on auditory perception and speech intelligibility in deaf children implanted younger than 18 months in comparison to those implanted at later ages. Otol Neurotol 2010;31:1261-7.

40. Stelmachowicz PG, Pittman AL, Hoover BM, Lewis DE, Moeller MP. The importance of high-frequency audibility in the speech and language development of children with hearing loss. Arch Otolaryngol Head Neck Surg 2004;130:556-62.

41. Choi HS, Choi JY, Moon IS, et al. Limitation of high pitch sound perception in nontumor patients with auditory brainstem implantation. Korean J Otorhinolaryngol Head Neck Surg 2017;5:235-41.

42. Goffi-Gomez MVS, Magalhães AT, Neto RB, Tsuji RK, Gomes MdQT, Bento RF. Auditory brainstem implant outcomes and MAP parameters: Report of experiences in adults and children. Int $J$ Pediatr Otorhinolaryngol 2012;76:257-64.

43. Vincent C. Auditory brainstem implants: How do they work? Anat Rec (Hoboken) 2012;295:1981-6.

44. Sanna M, Khrais T, Guida M, Falcioni M. Auditory brainstem implant in a child with severely ossified cochlea. Laryngoscope 2006;116:1700-3.

45. Yücel E, Aslan F, Özkan HB, Sennaroğlu L. Recent rehabilitation experience with pediatric ABI users. J Int Adv Otol 2015;11:110-3.

46. Geers AE, Sedey AL. Language and verbal reasoning skills in adolescents with 10 or more years of cochlear implant experience. Ear Hear 2011;32 (1 suppl):39S.

47. Nicholas JG, Geers AE. Will they catch up? The role of age at cochlear implantation in the spoken language development of children with severe to profound hearing loss. J Speech Lang Hear Res 2007;50:1048-62. 